\title{
Perfiles en hostilidad e impulsividad de una muestra de pacientes con trastorno límite de la personalidad en terapia dialectico-conductual.
}

\section{Hostility and Impulsivity profiles in a sample of patients with Borderline Personality Disorder receiving Dialectical Behavior Therapy.}

RESUMEN: Descripción y seguimiento de niveles de hostilidad e impulsividad de una muestra de pacientes en terapia dialécticoconductual grupal.

PALABRAS CLAVE: Trastorno límite de la personalidad, hostilidad, impulsividad, terapia Dialéctico-Conductual.
ABSTRACT: Levels of hostility and impulsivity in a sample of patients with Borderline Personality Disorder treated with DBT in a group setting. Description and follow up.

KEY WORDS: Borderline personality disorder, hostility, impulsivity, Dialectical Behaviour Therapy.

\section{Introducción.}

El Trastorno limite de la personalidad (TLP) es un trastorno complejo, caracterizado según los criterios diagnósticos del DSM IV por un patrón general de inestabilidad en las relaciones interpersonales, en la autoimagen y en la afectividad, y una notable impulsividad, que comienza al inicio de la edad adulta y se da en diversos contextos.

Es el trastorno de personalidad más frecuente (2\% de la población general), y además presenta una alta comorbilidad con trastornos afectivos, de ansiedad y de abuso de sustancias y una elevada tasa de suicidios (tasa de suicidio consumado 9\%), lo que en su conjunto provoca una gran demanda asistencial.

Diversos autores, en especial M. Linehan, han señalado la desregulación emocional como el elemento patogénico fundamental del trastorno límite de la personalidad, a partir del cual podrían explicarse los otros tres grupos de áreas problemáticas: impulsividad, el trastorno de identidad y el caos interpersonal.

Dentro de la desregulación emocional, distintas teorías (ej. Kernberg) han subrayado la centralidad del rol de la agresividad, y distintos estudios han relacionado altos niveles de agresividad o desregulaciones de la rabia con experiencias pasadas de apego desorganizadas o experiencias de abuso y/o violencia. Efectivamente, se ha comprobado que los pacientes afectos de TLP muestran una 
prevalencia significativamente mayor que la población general de experiencias de abuso/violencia y de patrones de apego desorganizado en la infancia. Además, altos niveles de hostilidad han sido relacionados con un aumento del nivel general de impulsividad.

Estas observaciones nos hacen pensar en la importancia de la evaluación y seguimiento de los niveles de hostilidad en estos pacientes, sea por su relación con los niveles de impulsividad sea como indicadores de sufrimiento en si mismo.

Por otra parte, la Terapia Dialéctico-Conductual se está afirmando en los últimos años como una de las terapias de elección para el tratamiento del TLP. Las Guías Clínicas de la Asociación Psiquiátrica Americana la recomiendan como un tratamiento de primera línea, y estudios internacionales están demostrando su eficacia en el tratamiento de las principales conductas disfuncionales presentes en este trastorno, especialmente de las conductas impulsivas, y en la adquisición de habilidades adaptativas de regulación emocional, especialmente de la rabia/hostilidad.

En este trabajo se presenta una muestra de pacientes diagnosticados de TLP que han sido derivados al Hospital de Día de Psiquiatría (HDDS) del Hospital Universitario Son Dureta para participar en la terapia Dialéctico-Conductual grupal, a quienes han sido evaluados y monitorizados los niveles de hostilidad y de impulsividad mediante la Escala de Impulsividad de Barratt (BIS-11) y el Inventario de Hostilidad de Buss-Durkee (BDHI).

\section{Objetivos.}

En este trabajo pretendemos:

- Trazar un perfil de las puntuaciones en hostilidad e impulsividad obtenidas mediante las escalas BDHI y BIS-11 de la muestra de pacientes derivadas al HDDS para el grupo de tratamiento para el TLP, prestando atención a las distintas subescalas y comparando las puntuaciones obtenidas en la muestra con los puntos de corte establecidos para la población general.

- Comparar las puntuaciones de las medidas pre y post tratamiento grupal, para valorar posibles cambios en los niveles de hostilidad e impulsividad en el periodo en el que los pacientes han estado en tratamiento.

\section{Método.}

La muestra del estudio está formada por 27 pacientes derivados desde dis- 
ORIGINALES Y REVISIONES

tintos servicios de salud mental del área del Hospital Son Dureta (Unidades de Salud Mental, Unidad de Trastornos de la Conducta Alimentaria, Centros de Atención a las Drogodependencias) para participar al grupo de terapia Dialéctico-Conductual para el tratamiento del trastorno límite de la personalidad.

A todos los participantes viene realizada una evaluación inicial pormenorizada, compuesta por entrevistas clínicas diagnósticas y tests clínicos específicos para evaluar eventuales comorbilidades.

En esta evaluación inicial se administra también la Escala de Hostilidad de Buss-Durkee (BDHI), actualmente la más usada para la valoración de los niveles de agresividad. Esta escala autoadministrada está formada por 75 ítems SI/NO y está compuesta por una puntuación total y 8 subescalas: Violencia, Hostilidad Indirecta, Irritabilidad, Negativismo, Resentimiento, Recelos, Hostilidad Verbal y Culpabilidad.

Se presentan a continuación las descripciones de las subescalas:

- Violencia: Mide la violencia física hacia los demás y la tendencia a enzarzarse en peleas. No incluye la violencia verbal ni la destrucción de objetos materiales.

- Hostilidad indirecta: Evalúa las conductas hostiles indirectas, como los chismorreos o bromas y la descarga de afecto negativo hacia los demás, sin centrarse en nadie en particular.

- Irritabilidad: Se refiere a la tendencia a explotar en reacciones de afecto negativo ante pequeñas provocaciones. Incluye además el malhumor constante, la exasperación y la rudeza.

- Negativismo: Se refiere a una conducta oposicionista generalizada hacia la autoridad. Implica rechazo a la cooperación, ya sea en forma de incumplimientos pasivos o de rebelión manifiesta hacia las convenciones o normas.

- Resentimiento: Evalúa los celos y el odio hacia los demás. Incluye sentimientos de ira hacia el mundo, basados en malos tratos reales o imaginarios.

- Recelos: También denominada suspicacia o sospecha. Puede variar desde la mera desconfianza y cautela ante la gente hasta la creencia de que los demás son despectivos o planean el mal.

- Hostilidad Verbal: Se refiere a la expresión del afecto negativo mediante el habla, ya sea en su contenido o en su estilo. El estilo comprende conductas como dar voces y chillidos. El contenido puede incluir amenazas, insultos e hipercriticismo.

Además, los análisis factoriales han revelado la existencia de dos factores subyacentes a las subescalas: un componente experiencial, definido principalmente por las subescalas Resentimiento y Recelos, y un componente expresivo, definido por las subescalas Violencia, Hostilidad indirecta, Irritabilidad, Negativismo y Hostilidad Verbal. 
Asimismo se administra la Escala de Impulsividad de Barratt (BIS-11), uno de los instrumentos más usados para la valoración de la impulsividad. Se trata de una escala autoadministrada de 30 cuestiones, compuesta por una puntuación total y 3 subescalas: Impulsividad Cognitiva, Impulsividad Motora e Impulsividad no Planeada.

Se presentan a continuación las descripciones de las subescalas:

- Impulsividad Cognitiva: se refiere principalmente a la tendencia a reflexionar de forma poco cuidadosa y a tomar decisiones rápidamente.

- Impulsividad Motora: mide la propensión a actuar rápidamente o guiado principalmente por los estímulos presentes en el momento. Está relacionado con una escasa mediación cognitiva y con una baja capacidad de autocontrol.

- Impulsividad no Planeada: evalúa la planificación y organización de las actuaciones futuras.

La evaluación post-tratamiento se realiza una vez acabada la terapia grupal, aproximadamente después de unos 2 años.

\section{Resultados.}

Perfil de puntuaciones pre

Del total de la muestra, se pudo administrar la escala BDHI a 24 de los pacientes, y la escala BIS-11 a 26 de ellos.

Se presentan a continuación algunos datos descriptivos (media y desviación típica) de la muestra, junto con los puntos de corte establecidos para discriminar población general y clínica de las escalas BDHI y BIS-11. (Tabla 1)

Perfil puntuaciones post

Del total de la muestra, actualmente 6 pacientes han finalizado el tratamiento.

Se presentan a continuación algunos datos descriptivos (media y desviación típica) de estos pacientes, junto con los puntos de corte establecidos para discriminar población general y clínica de las escalas BDHI y BIS-11. (Tabla 2).

Diferencias entre las puntuaciones pre y post

Para los 6 sujetos que finalizaron el tratamiento se ha realizado la comparación de sus puntuaciones en hostilidad e impulsividad antes y después del tratamiento, siempre teniendo en cuenta que, por distintas razones entre las cuales el tamaño de la muestra y el diseño del estudio, no se pueden sacar conclusiones relativas a la eficacia de la intervención Dialéctico-Conductual, sino solamente una mera descripción de la presencia o ausencia de cambios significativos.

Se presentan a continuación los datos obtenidos. (Tabla 3, Gráfico 1, Gráfico 2). 
ORIGINALES Y REVISIONES

Descripción del perfil de la muestra en hostilidad e impulsividad

De los datos y gráficos obtenidos podemos realizar las siguientes observaciones:

1-Las puntuaciones totales de nuestra muestra en las escalas BIS-11 y BDHI superan ampliamente los puntos de corte establecidos para distinguir población clínica y no clínica.

2- Las puntuaciones de la muestra en todas las subescalas se encuentran por encima de los puntos de corte, exceptuando la subescala Hostilidad Indirecta. Las subescalas Culpabilidad y Negativismo no se han tenido en cuenta debido a la ausencia de puntos de corte establecidos.

3-Las subescalas que han presentado una mayor puntuación con respecto a los puntos de corte han resultado Impulsividad Motora en la escala BIS-11 y Resentimiento y Recelos en la escala BDHI.

De estas tres observaciones podemos concluir que nuestra muestra está caracterizada por altos niveles generales de hostilidad y de impulsividad, puntuaciones que superan ampliamente los niveles de la población general. Este fenómeno se repite a su vez en todas las subescalas, exceptuando Hostilidad Indirecta. Podríamos hipotetizar que este resultado es debido a que los niveles de hostilidad vienen expresados de manera más directa y franca (véase las altas puntuaciones en las subescalas Violencia y Hostilidad Verbal).

Por otra parte, nos parecen llamativas las puntuaciones de nuestra muestra en las subescalas Resentimiento y Recelos. Estas subescalas, que definen el componente experiencial de la hostilidad, han resultado ser las más elevadas con respecto a los puntos de corte establecidos para la población, indicándonos que nuestra muestra está caracterizada por niveles elevados de hostilidad en su vertiente expresada pero por niveles aún más elevados de hostilidad en su vertiente experiencial. Atendiendo a la literatura científica sobre este trastorno se podría plantear la relación entre estos altos niveles de hostilidad experiencial con las altas tasas de experiencias pasadas de violencia y malos tratos que caracteriza nuestra muestra.

Comparacion de las medidas pre y post tratamiento

En cuanto a las puntuaciones en la escala BDHI:

1- Se han observado puntuaciones menores en todas las medidas post respecto a las medidas pre, tanto en la escala total como en las subescalas.

2-Las mayores disminuciones se han observado en las subescalas Hostilidad Verbal, Resentimiento y Hostilidad Total.

3-Dos de estas disminuciones han resultado significativas: las relativas a las escalas Hostilidad Verbal y Escala Total.

4- En el caso de la escala Hostilidad Verbal se ha observado además que la puntuación post se encuentra por debajo del punto de corte para la población no clínica. 
En cuanto a las puntuaciones en la escala BIS-11:

1-Se han observado reducciones de las medidas post en las subescalas impulsividad motora e impulsividad total.

2- Estas disminuciones no han resultado significativas.

De estas observaciones podemos concluir que en el periodo en el que los pacientes han estado en tratamiento ha habido una disminución significativa del nivel general de hostilidad, principalmente en la faceta de hostilidad verbal. La mayor parte de la reducción se observa por tanto en la vertiente expresiva de la hostilidad, aunque en la vertiente experiencial se han observado también mejorías.

Por otra parte, se ha observado una cierta reducción de los niveles de impulsividad total y motora, aunque esta reducción no ha resultado significativa.

\section{Conclusiones y Discusión}

Los datos obtenidos nos han indicado que el perfil de nuestra muestra está caracterizado por altos niveles de hostilidad y de impulsividad, todos (excepto la subescala hostilidad indirecta) por encima de los puntos de corte establecidos para la distinción de la población clínica y no clínica. Las subescalas relativas al componente experiencial de la hostilidad han resultado ser las más elevadas.

En cuanto a la comparación de los niveles de hostilidad e impulsividad antes y después del tratamiento, los datos nos indican una reducción de todas las puntuaciones en hostilidad en las medidas post tratamiento, y una reducción de los niveles de impulsividad en la escala total y en la subescala impulsividad motora. De estas reducciones, las relativas a la escala de hostilidad verbal y hostilidad total han resultado significativas.

Sin embargo, el diseño del estudio y el tamaño de la muestra no nos permiten sacar conclusiones en cuanto al papel causal del tratamiento grupal en estas mejorías clínicas, por lo que nuestras conclusiones se limitan a un nivel descriptivo. 
Tabla 1

\begin{tabular}{l|c|c|c}
\hline \multicolumn{1}{c|}{ Variable } & Media & Desviación Típica & Punto de Corte \\
\hline BDHI Esc Total PRE & 45,42 & 10,021 & 27 \\
\hline Violencia & 4,42 & 2,358 & 3 \\
\hline Hostilidad & 5,13 & 1,329 & 6 \\
\hline Irritabilidad & 7,17 & 1,903 & 2 \\
\hline Negativismo & 3,38 & 1,439 & NO ESPECIFICADO \\
\hline Resentimiento & 5,17 & 1,373 & 6 \\
\hline Recelos & 5,79 & 1,956 & \\
\hline Hostilidad verbal & 8,29 & 1,654 & NO ESPECIFICADO \\
\hline Culpabilidad & 5,96 & 2,136 & \\
\hline & & & 32,5 \\
\hline & & & 9,5 \\
\hline BIS 11 Total & 65,35 & 16,98 & 9,5 \\
\hline BIS 11 Imp cognitiva & 19,38 & 4,54 & 14 \\
\hline BIS 11 Imp Motora & 24,5 & & \\
\hline BIS 11 Imp No planeada & 21,46 & 6,67 & $\mathrm{~N}=26$ \\
\hline
\end{tabular}

Tabla 2

\begin{tabular}{l|c|c|c}
\hline \multicolumn{1}{c|}{ Variable } & Media & Desviación Típica & Punto de Corte \\
\hline BDHI Esc total POST & 35,33 & 10,764 & 27 \\
\hline Violencia post & 3,83 & 3,251 & 3 \\
\hline Hostilidad post & 4,33 & 1,751 & 6 \\
\hline Irritabilidad post & 6,67 & 1,751 & 6 \\
\hline Negativismo post & 3,00 & 1,789 & NO ESPECIFICADO \\
\hline Resentimiento post & 3,17 & 1,602 & 2 \\
\hline Recelos post & 4,83 & 2,787 & 6 \\
\hline Hostilidad Verbal post & 5,83 & 1,472 & NO ESPECIFICADO \\
\hline Culpabilidad post & 3,67 & 1,033 & 32,5 \\
\hline & & & 9,5 \\
\hline BIS-11 Total POST & 60,33 & 17,24 & 9,5 \\
\hline BIS-11 Imp Cogn Post & 19,67 & 5,05 & 14 \\
\hline BIS-11 Imp Mot Post & 19,83 & 5,71 & \\
\hline BIS-11 Imp No Plan Post & 20,5 & 8,55 & N $=6$ \\
\hline
\end{tabular}


Tabla 3

\begin{tabular}{l|c|c|c|c|c|c}
\hline Variable Pre-Post & Media & $\begin{array}{c}\text { Desviación } \\
\text { Típica }\end{array}$ & $\begin{array}{c}\text { Intervalo de } \\
\text { Confianza para la } \\
\text { diferencia 95\% }\end{array}$ & $\begin{array}{c}\text { Estadístico t } \\
\text { (5 grados de } \\
\text { libertad) }\end{array}$ & $\begin{array}{c}\text { Significabilidad } \\
\text { bilateral }\end{array}$ & \\
\hline BDHI Total & 8,333 & 6,346 & $1,674-14,993$ & 3,217 & 0,024 & $*$ \\
\hline Violencia & 1,167 & 2,137 & $-1,076-3,409$ & 1,337 & 0,239 & \\
\hline Hostilidad & 0,167 & 1,722 & $-1,641-1,974$ & 0,237 & 0,822 & \\
\hline Irritabilidad & 1,333 & 1,751 & $-0,504-3,171$ & 1,865 & 0,121 & \\
\hline Negativismo & 0,333 & 0,816 & $-0,524-1,190$ & 1,000 & 0,363 & \\
\hline Resentimiento & 0,833 & 1,472 & $-0,711-2,378$ & 1,387 & 0,224 & \\
\hline Recelos & 0,333 & 1,033 & $-0,751-1,417$ & 0,791 & 0,465 & \\
\hline Hostilidad verbal & 2,333 & 0,816 & $1,476-3,190$ & 7,000 & 0,001 & $*$ \\
\hline Culpabilidad & 1,833 & 2,229 & $-0,505-4,172$ & 2,015 & 0,100 & \\
\hline BIS-11 Total & & & & & & \\
\hline BIS-11 Imp Cogn & $-2,67$ & 5,16 & $-8,086-2,753$ & $-1,27$ & 0,62 & \\
\hline BIS-11 Imp Mot & $-0,5$ & 8,55 & $-9,473-8,473$ & $-0,14$ & 0,89 & \\
\hline BIS-11 Imp No Plan & 0 & 6,1 & $-6,401-6,401$ & 0 & & 1 \\
\hline
\end{tabular}

Gráfico 1

Comparación puntuaciones BDHI previas al tratamiento, posteriores al tratamiento y puntos de corte para discriminar población general y clínica

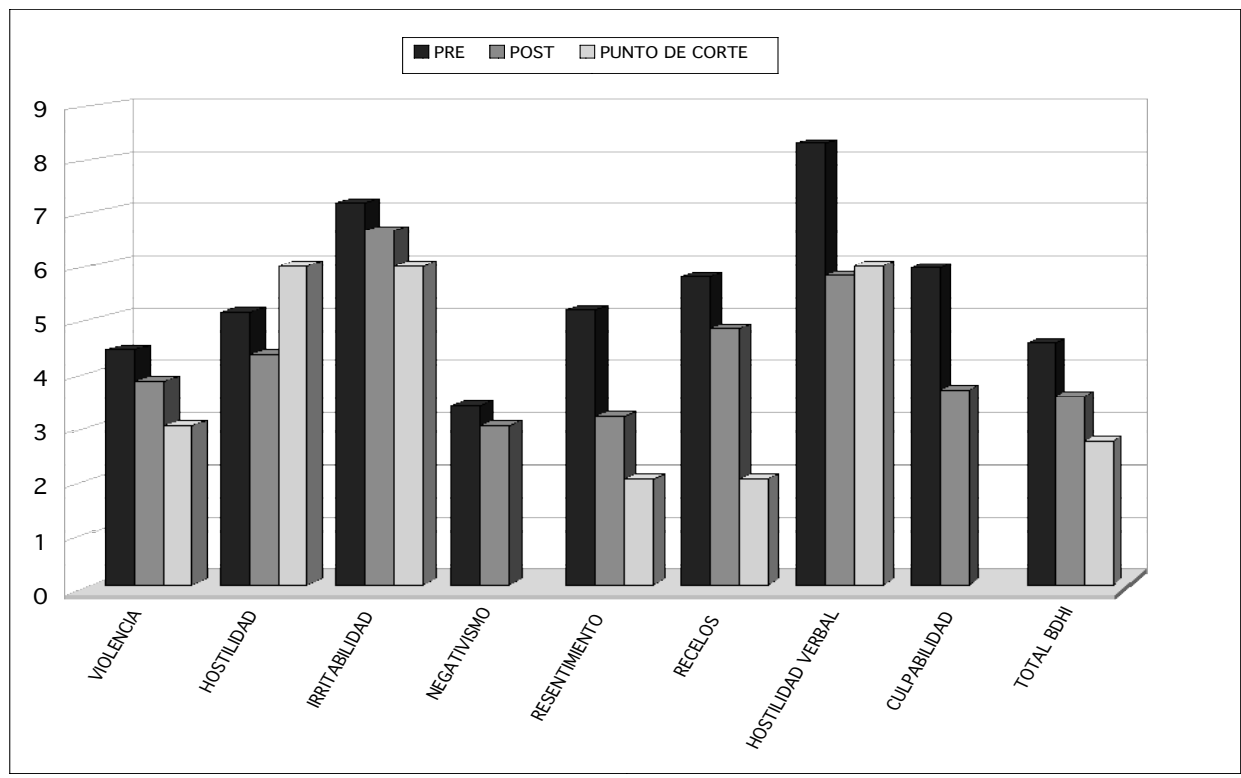


Gráfico 2

Comparación puntuaciones BIS-11 previas al tratamiento, posteriores al tratamiento y puntos de corte para discriminar población general y clínica

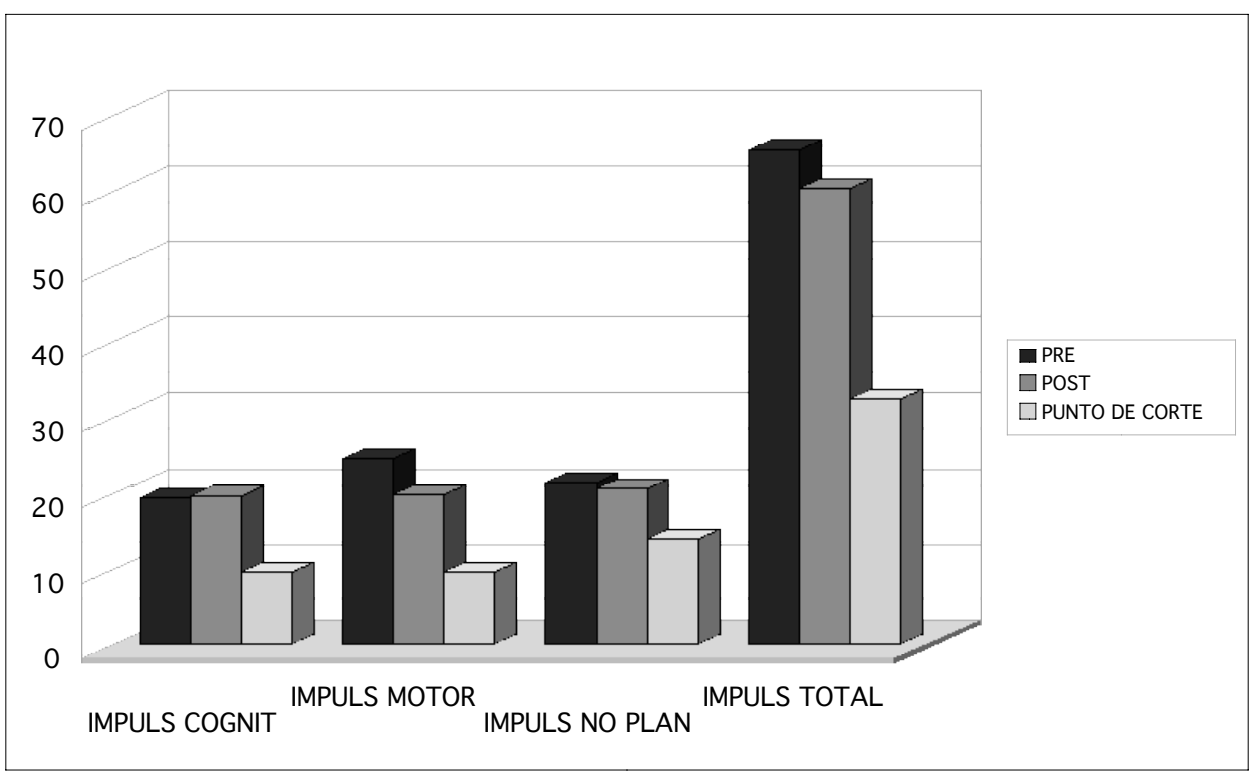


ORIGINALES Y REVISIONES

\section{BIBLIOGRAFÍA:}

(1) LóPez-Ibor Aliño, J. ; Valdés Miyar, M. (DIR.) DSM-IV-TR. Manual diagnóstico y estadístico de los trastornos mentales. Texto revisado. Barcelona, Masson, 2002

(2) Linehan, M. M. Manual del tratamiento de los trastornos de personalidad límite, lugar de la edición, Paidos Iberica, año.

(3) M.P. García-Portilla, y otros, Banco de Instrumentos Básicos para la práctica de la psiquiatría clínica. Ed 4º Lugar de edición, Ars Médica. 2006.

(4) OQuendo MA, Y otros. "Spanish adaptation of Buss-Durkee Hostility Inventory (BDHI)". Eur J Psychiatry, 2001, 15, 101-112

(5) OQuendo MA, y Otros. "Spanish adaptation of the Barratt Impulsiveness Scale (BIS)". Eur J Psychiatry, 2001, 15, 147-155

(6) Feigenbaum J. "Dialectical behaviour therapy: An increasing evidence base". Journal of Menthal Health, 2007, 16(1), 51-68

(7) Evershed S., Y OTROs. "Practice-based outocomes of dialectical behaviour therapy (DBT) targeting anger and violence, with male forensic patients: a pragmatic and non-contemporaneous comparison". Criminal Behaviour and Mental Health, 2003, 13, 198-213

(8) Agrawal H.R., y otros. "Attachment Studies with Borderline Patients: A Review". Harvard Review of Psychiatry, 2004, vol.12, n²

(9) Critchileld K.L., y otros. "The Relational Context of Aggression in Borderline Personality Disorder: Using Adult Attachment Style to Predict Forms of Hostility". Journal of Clinical Psychology, 2008, Vol.64(1), 67-82

\footnotetext{
* Maria Acuñas Gelabert. Residente de Psicología Clínica. Hospital Universitario Son Dureta. Carmen Bermúdez de la Puente Andión. Residente de Psicología Clínica. Hospital Universitario Son Dureta. Eva Lago Blanco. Residente de Psicología Clínica. Hospital Universitario Son Dureta. Andrea Vilavedra Vaamonde. Residente de Psicología Clínica. Hospital Universitario Son Dureta. Noelia Marín Sánchez. Psicóloga Clínica. Hospital Universitario Son Dureta. Olga Ibarra Uría. Psiquiatra. Hospital Universitario Son Dureta.
}

Correspondencia: Correo electrónico: maria_acunas@yahoo.com

** Recibido: 26/05/2009 